\title{
Riemannian Graph Diffusion for DT-MRI Regularization
}

\author{
Fan Zhang and Edwin R. Hancock \\ Department of Computer Science, University of York, York, YO10 5DD, UK
}

\begin{abstract}
A new method for diffusion tensor MRI (DT-MRI) regularization is presented that relies on graph diffusion. We represent a DT image using a weighted graph, where the weights of edges are functions of the geodesic distances between tensors. Diffusion across this graph with time is captured by the heat-equation, and the solution, i.e. the heat kernel, is found by exponentiating the Laplacian eigen-system with time. Tensor regularization is accomplished by computing the Riemannian weighted mean using the heat kernel as its weights. The method can efficiently remove noise, while preserving the fine details of images. Experiments on synthetic and real-world datasets illustrate the effectiveness of the method.
\end{abstract}

\section{Introduction}

Diffusion tensor MRI is an important tool for non-invasive exploration of the anatomic structure of the white matter in vivo. It endows each voxel with a $3 \times 3$ symmetric positive-definite matrix, which measures the anisotropic behavior of water diffusion in white matter. Due to the motion of the ventricles and the partial volume effect, DT images are often noisy. Thus, regularization is necessary before fiber tracking can commence. Because of the high dimensionality of the tensor data and constraints imposed by the curved feature space of tensors, traditional scalar image smoothing techniques are often no longer applicable. Generally, tensor field regularization can be realized in one of the three ways: smoothing the whole tensors [12, separately smoothing the eigenvectors and eigenvalues of tensors 345, and directly smoothing the diffusion weighted images before estimation of the tensor [6].

The aim of this paper is to show how the heat kernel can be used to develop a graph-spectral method for tensor field regularization by working on the Riemannian feature space of tensors. Unlike prior literature on image smoothing using continuous partial differential equations (PDEs), our method is based on the heat equation for discrete structures [7. We represent tensor-valued images as weighted undirected graphs, where the edge weights are functions of geodesic distances between tensors. Diffusion across this weighted graph with time is captured by the heat-equation, and the solution, i.e. the heat kernel, is found by exponentiating the Laplacian eigen-system with time. Diffusion tensor image regularization is accomplished by convolving the heat kernel with the image, 
which is equal to the Riemannian weighted mean due to the fact that tensors are points on a Riemannian manifold 8922.

The next section of this paper summarises the Riemannian space of the tensors, and defines the geodesic distance and Riemannian weighted mean on it, which are necessary for the main algorithm. Section 3 proposes the new graph diffusion-based method for tensor field regularization. Experiments on synthetic and real DT-MRI data are shown in Section 4. Section 5 concludes the paper.

\section{Riemannian Space of Tensors}

Let $\Sigma(r)$ be the set of $r \times r$ real matrices. Recall that in $\Sigma(r)$ the Euclidean inner products, which is known as the Frobenius inner product, is defined as $\langle A, B\rangle_{F}=\operatorname{tr}\left(A^{T} B\right)$, where $\operatorname{tr}(\cdot)$ denotes the trace and superscript $T$ denotes the transpose. For a matrix $A$ whose eigen-decomposition is $A=U \Lambda U^{T}$, the exponential of $A$ is given by $\exp A=\sum_{k=0}^{\infty} \frac{1}{k !} A^{k}=U \exp (\Lambda) U^{T}$, and the inverse logarithm of $A$ is given by $\log A=-\sum_{k=1}^{\infty} \frac{(I-A)^{k}}{k}=U \log (\Lambda) U^{T}$, where $I$ is the identity matrix.

Let $S(r)$ be the space of $r \times r$ symmetric matrices and $S^{+}(r)$ be the space of symmetric positive-definite matrices. Thus, the space $M$ of diffusion tensors is identified with $S^{+}(3)$. Through the identity mapping $\psi: P \in S^{+}(r) \rightarrow$ $\left(\sigma_{11}, \ldots, \sigma_{i j}\right), \quad i \leq j, S^{+}(r)$ is isomorphic with an open subset $\Omega$ of the $R^{m}$ where $m=\frac{1}{2} r(r+1)$. Thus we could consider $S^{+}(r)$ as a $m$-dimensional differential manifold with $(\Omega, \psi)$ as the coordinate system. At each point $P \in S^{+}(r)$ the tangent space $T_{P} S^{+}(r)$ is equal to $S(r)$. So a basis of $T_{P} S^{+}(r)$ can be defined as $\frac{\partial}{\partial \sigma_{i j}} \leftrightarrow \Pi_{i j} \in S(r), \quad i \leq j$, where $\Pi_{i j}=1_{i i}$ if $i=j, \Pi_{i j}=1_{i j}+1_{j i}$ if $i \neq j$, and $1_{i j}$ means the $r \times r$ matrix with 1 at element $(i, j)$ and 0 elsewhere.

We can turn $S^{+}(r)$ into a Riemannian manifold by introducing a Riemannian metric $g$ at $P$ [10], i.e. $g\left(\frac{\partial}{\partial \sigma_{i j}}, \frac{\partial}{\partial \sigma_{k l}}\right)=g\left(\Pi_{i j}, \Pi_{k l}\right)=\operatorname{tr}\left(P^{-1} \Pi_{i j} P^{-1} \Pi_{k l}\right)$. This is the same as the affine-invariant inner product used by [8112], i.e. $\langle A, B\rangle_{P}=$ $\operatorname{tr}\left(P^{-1} A P^{-1} B\right), A, B \in T_{P} S^{+}(r)$. Thus, for a smooth curve $C:[a, b] \rightarrow$ $S^{+}(r)$, the length of $C(t)$ can be computed via the invariant metric, i.e. $\ell(C)=$ $\int_{a}^{b}\left\|C^{\prime}(t)\right\|_{C(t)}=\int_{a}^{b} \sqrt{\operatorname{tr}\left(C(t)^{-1} C^{\prime}(t)\right)^{2}}$. The distance between two points $A, B \in$ $S^{+}(r)$ is the infimum of lengths of curves connecting them, i.e. $d(x, y):=\underset{C}{\operatorname{argmin}}$

$\{\ell(C) \mid C(a)=A, C(b)=B\}$. The curve satisfying this infimum condition is a geodesic. In $S^{+}(r)$ the geodesic with initial point at identity matrix $I$ and tangent vector $X \in T_{I} S^{+}(r)$ is given by $\exp (t X)$. Using invariance under group actions [11, an arbitrary geodesic $\Gamma(t)$ such that $\Gamma(0)=P$ and $\Gamma^{\prime}(0)=X$ is given by $\Gamma_{(P, X)}(t)=P^{\frac{1}{2}} \exp \left(t P^{-\frac{1}{2}} X P^{-\frac{1}{2}}\right) P^{\frac{1}{2}}$. Thus, the geodesic distance between two points $A$ and $B$ in $S^{+}(r)$ is

$$
d_{g}(A, B)=\left\|\log \left(A^{-1} B\right)\right\|_{F}=\sqrt{\sum_{i=1}^{n}\left(\log \lambda_{i}\right)^{2}},
$$

where $\lambda_{i}$ are the eigenvalues of $A^{-1} B$. 
We can relate an open subset of the tangent space $T_{P} S^{+}(r)$ with a local neighborhood of $P$ in $S^{+}(r)$ using the exponential map $\operatorname{Exp}: \Omega \subset T_{P} S^{+}(r) \rightarrow$ $S^{+}(r)$, which is defined as $\operatorname{Exp}_{P}(X)=\gamma_{(P, X)}(1)$. Geometrically, $\operatorname{Exp}_{P}(X)$ is a point of $S^{+}(r)$ obtained by marking out a length equal to $|X|$ commencing from $P$, along a geodesic which passes through $P$ with velocity equal to $\frac{X}{|X|}$. From equation 2, it follows

$$
\operatorname{Exp}_{P}(X)=P^{\frac{1}{2}} \exp \left(P^{-\frac{1}{2}} X P^{-\frac{1}{2}}\right) P^{\frac{1}{2}} .
$$

Since $\operatorname{Exp}_{P}$ is a local diffeomorphism, it has an inverse map, the so-called logarithmic map $\log _{P}: S^{+}(r) \rightarrow B_{\epsilon}(0) \subset T_{P} S^{+}(r)$ where $\log _{P}\left(\gamma_{(P, X)}(t)\right)=t X$. Thus, for a point $A$ near $P$ it also follows

$$
\log _{P}(A)=P^{\frac{1}{2}} \log \left(P^{-\frac{1}{2}} A P^{-\frac{1}{2}}\right) P^{\frac{1}{2}} .
$$

\subsection{Riemannian Weighted Mean}

Consider a set of $n$ points (or tensors) $p_{1}, \ldots, p_{n}$ residing on the Riemannian manifold $M=S^{+}(3)$ of tensors, with corresponding weights $a_{1}, \ldots, a_{n} \in R$ such that $\sum_{k=1}^{n} a_{k}=1$. An efficient way of characterising the points is to compute their weighted mean. If the points are in $\Sigma(3)$, recall that the arithmetic Euclidean weighted mean $q$ can be defined as the linear combination $q=\sum_{k=1}^{n} a_{k} p_{k}$, From a variational standpoint the Euclidean weighted mean minimizes the sum of the weighted squared distances $f(q)$ to the given points $p_{k}$, i.e. $f(q)=\frac{1}{2} \sum_{k=1}^{n} a_{k} d_{e}\left(q, p_{k}\right)^{2}$ where $d_{e}\left(q, p_{k}\right)=\left\|q-p_{k}\right\|$ is the Euclidean distance between $q$ and $p_{k}$.

To generalise the least squares minimization to the manifold $M$, one intuitive way is to replace $d_{e}\left(q, p_{k}\right)$ by the shortest geodesic distance $d_{g}\left(q, p_{k}\right)$ on $M$ between the mean $q$ and the point $p_{k}$. Thus, we can define the Riemannian weighted mean as the point $q$ that minimizes the sum of geodesic distances $f(q)=\frac{1}{2} \sum_{k=1}^{n} a_{k} d_{g}\left(q, p_{k}\right)^{2}$. The mean $q$ is a critical point of $f$, i.e. the location where the directional first derivatives of $f$ are all zero. To locate the minimum of $f$, one widely used and easy to implement numerical method is to use steepest gradient descent 892 . At a point $q \in M$, the gradient of $f$ with respect to $q$ is $\nabla f(q)=-\sum_{k=1}^{n} a_{k} \log _{q}\left(p_{k}\right)$. The minimum of $f$ is located at the critical point $q, \nabla f(q)=0$, where the Euclidean weighted mean of tangent vectors $\log _{q}\left(p_{k}\right) \in T_{q} M$ is zero. The iteration of the classical gradient descent method can be stated as $q^{t+1}=q^{t}-\nabla f$. This iteration scheme means that the estimated mean $q^{t+1}$ moves towards mean $q$ in the direction of steepest descent. Thus, the intrinsic gradient descent algorithm on $M$ gives the following iteration scheme for estimating the Riemannian weighted mean of the tensor set $p_{1}, \ldots, p_{n}$,

$$
q^{t+1}=\operatorname{Exp}_{q^{t}}\left(\sum_{k=1}^{n} a_{k} \log _{q^{t}}\left(p_{k}\right)\right),
$$

where Exp and Log operators are defined in Eq. 2 and Eq. 3 respectively. 


\section{Graph Tensor Regularization}

Let $\Re$ be the diffusion tensor image under regularization. Following recent work on graph-spectral methods for image segmentation [12] we abstract a tensorvalued image using a weighted graph $G=(V, E, w)$, where the nodes $V$ of the graph are the voxels of the image $\Re$, and an edge is formed between every pair of nodes. The weight of each edge, $w(i, j) \in[0,1]$, is a function characterizing the relationship between the voxels $i$ and $j$.

Since we wish to adopt a graph-spectral approach, we introduce the weighted adjacency matrix $W$ for the graph where the elements are $W(i, j)=w(i, j)$ if $(i, j) \in E$, and $W(i, j)=0$ otherwise. We also construct the diagonal degree matrix $D$, whose elements are given by $D(i, i)=\operatorname{deg}(i)=\sum_{j \in V} W(i, j)$. From the degree matrix and the weighted adjacency matrix we construct the Laplacian matrix $L=D-W$, i.e. the degree matrix minus the weighted adjacency matrix. The spectral decomposition of the Laplacian matrix is $L=\Phi \Lambda \Phi^{T}$ where $\Lambda=\operatorname{diag}\left(\lambda_{1}, \lambda_{2}, \ldots, \lambda_{|V|}\right)$ is the diagonal matrix with the eigenvalues ordered according to increasing magnitude $\left(0=\lambda_{1}<\lambda_{2} \leq \lambda_{3} \ldots\right)$ as elements and $\Phi=\left(\phi_{1}\left|\phi_{2}\right| \ldots . \mid \phi_{|V|}\right)$ is the matrix with the correspondingly ordered eigenvectors as columns. Since $L$ is symmetric and positive semi-definite, the eigenvalues of the Laplacian are all positive. The eigenvector $\phi_{2}$ associated with the smallest non-zero eigenvalue $\lambda_{2}$ is referred to as the Fiedler-vector.

\subsection{Heat Kernel on Graphs}

We here introduce the heat equation associated with the Laplacian [13], i.e.

$$
\frac{\partial h_{t}}{\partial t}=-L h_{t}
$$

where $h_{t}$ is the heat kernel and $t$ is time. The heat-kernel satisfies the initial condition $h_{0}=I_{|V|}$ where $I_{|V|}$ is the $|V| \times|V|$ identity matrix.

The heat kernel can hence be viewed as describing the flow of heat across the edges of the graph with time. The rate of flow is determined by the Laplacian of the graph. The solution to the heat equation is found by exponentiating the Laplacian eigen-spectrum, i.e.

$$
h_{t}=e^{-t L}=\Phi e^{-t \Lambda} \Phi^{T} .
$$

The heat kernel is a $|V| \times|V|$ matrix, and for the nodes $i$ and $j$ of the graph $G$ the resulting element is $h_{t}(i, j)=\sum_{i=1}^{|V|} e^{-\lambda_{i} t} \phi_{i}(i) \phi_{i}(j)$. When $t$ tends to zero, then $h_{t} \simeq I-L t$, i.e. the kernel depends on the local connectivity structure or topology of the graph. If, on the other hand, $t$ is large, then $h_{t} \simeq e^{-t \lambda_{2}} \phi_{2} \phi_{2}^{T}$, where $\lambda_{2}$ is the smallest non-zero eigenvalue and $\phi_{2}$ is the associated eigenvector, i.e. the Fiedler vector. Hence, the large time behavior is governed by the global structure of the graph. 


\subsection{Regularization as Graph Diffusion}

It is useful to consider the following picture of the heat diffusion process on graphs. Suppose that we inject a unit amount of heat at the node $k$ of a graph, and allow the heat to diffuse through the edges of the graph. The rate of diffusion along the edge $E(i, j)$ is determined by the edge weight $w(i, j)$. At time $t$, the value of the heat kernel $h_{t}(k, j)$ can be interpreted as the amount of heat accumulated at node $j$.

We would like to regularise the tensor image $\Re$ using the heat flow on graph $G$. To do this we inject at each node of $G$ an amount of heat energy equal to the tensor of the associated voxel. The heat at each node diffuses through the graph edges as time $t$ progresses. The edge weight plays the role of thermal conductivity. If two voxels belong to the same region, then the associated edge weight is large. As a result heat can flow easily between them. On the other hand, if two voxels belong to different regions, then the associated edge weight is very small, and hence it is difficult for heat to flow from one region to another. From the viewpoint of geometry, tensors are points of the Riemannian manifold $M=S^{+}(3)$. The above process means we let the tensor points move according to its local neighbors. We believe that the difference between two tensor points belonging to the same region is a result of noise only. We therefore, want them to move toward each other. Contrarily, if two tensor points belong to different regions, we want them stay where they are. The prior similarity between two voxels of $\Re$ is expressed in terms of the edge weight of $G$, which depends on two factors: 1). The geodesic distances between the tensors. 2). The Euclidean distances between the locations of the voxels. We encode all the tensors associated with the voxels of the image $\Re$ as a block column vector $\mathcal{I}_{0}$, whose elements $\mathcal{I}_{0}(i)$ are $3 \times 3$ tensors. We would like to minimize the influence of one region on another. This behavior is captured by the weight matrix

$$
W(i, j)= \begin{cases}e^{-\frac{d_{g}\left(\mathcal{I}_{0}(i), \mathcal{I}_{0}(j)\right)^{2}}{\kappa_{\mathcal{I}}^{2}}} * e^{-\frac{d_{e}(X(i)-X(j))^{2}}{\kappa_{X}^{2}}} & \text { if } d_{e}(X(i)-X(j)) \leq r \\ 0 & \text { otherwise }\end{cases}
$$

where the symbols used are as follows: $X(i)$ is the location of the voxel $i$; $d_{e}(X(i)-X(j))$ is the Euclidean distance between voxels $i$ and $j ; d_{g}\left(\mathcal{I}_{0}(i), \mathcal{I}_{0}(j)\right)$ is the geodesic distance between tensors $\mathcal{I}_{0}(i)$ and $\mathcal{I}_{0}(j)$, i.e. $d_{g}\left(\mathcal{I}_{0}(i), \mathcal{I}_{0}(j)\right)=$ $\left\|\log \left(\mathcal{I}_{0}(i)^{-1} \mathcal{I}_{0}(j)\right)\right\|_{F}$, as defined in Eq. 1, The values of $\kappa_{\mathcal{I}}$ and $\kappa_{X}$ are usually set to $10 \% \sim 20 \%$ of the total range of the distance functions $d_{g}$ and $d_{e}$. They has the effect of controlling the velocity of the diffusion process.

The heat diffusion process described above is governed by the same PDE as Eq. 5. however the initial conditions are different. Now the initial heat residing at each node is determined by the corresponding tensor. The evolution of the tensors $\mathcal{I}_{0}$ of image $\Re$ follows the equation

$$
\frac{\partial \mathcal{I}_{t}}{\partial t}=-L \mathcal{I}_{t}
$$

In fact, if we view each tensor is composed of six independent components, the above diffusion equation comprises a system of six coupled PDEs. The coupling 
results from the fact that the edge weight of $G$ depends on all the components of the tensors. The solution of Eq. 8 is $\mathcal{I}_{t}=e^{-t L} \mathcal{I}_{0}=h_{t} \mathcal{I}_{0}$. As a result the smoothed tensor of the voxel $j$ at time $t$ is

$$
\mathcal{I}_{t}(j)=\sum_{i=1}^{|V|} \mathcal{I}_{0}(i) \times h_{t}(i, j) .
$$

This is a measure of the total energy to flow from the remaining nodes to node $j$ during the elapsed time $t$. When $t$ is small, we have $\mathcal{I}_{t} \simeq(I-L t) \mathcal{I}_{0}$. Each row $i$ of the heat kernel $h_{t}$ satisfies the conditions $0 \leq h_{t}(i, j) \leq 1$ for $\forall j$ and $\sum_{j=1}^{|V|} h_{t}(i, j)=1$. Due to the constraints of the tensors $\mathcal{I}_{0}(i)$ imposed by the Riemannian space $M=S^{+}(3)$, the smoothed tensors $\mathcal{I}_{t}(j)$ should always stay on the manifold $M$. According to section 2.1, $\mathcal{I}_{t}(j)$ in Eq. 9 is the Riemannian weighted mean of the tensor set $\left\{\mathcal{I}_{0}(i)|i=1, \ldots| V \mid,\right\}$. The Riemannian weighted mean has the heat kernel as its weight set $\left\{h_{t}(i, j)|i=1, \ldots| V \mid,\right\}$. Thus, $\mathcal{I}_{t}(j)$ for voxel $j$ at time $t$ can be easily found by the intrinsic gradient descent, as stated in Eq. 4, i.e.

$$
\mathcal{I}_{t}(j)^{k+1}=\operatorname{Exp}_{\mathcal{I}_{t}(j)^{k}}\left(\sum_{i=1}^{|V|} h_{t}(i, j) \log _{\mathcal{I}_{t}(j)^{k}}\left(\mathcal{I}_{0}(i)\right)\right),
$$

where Exp and Log operators on $M$ are defined in Eq. 2 and Eq. 3 respectively.

\section{Experiments}

We have applied our Riemannian graph-spectral filter to synthetic and real-world tensor-valued images. In top row of Fig. 1] we generate a tensor field with three different regions, and add IID additive noise to eigenvectors and eigenvalues of the tensors respectively. As the figure shows, our graph diffusion well restore the coherence of the the field inside each structure without destroying the interfaces between regions. In order to better evaluate our algorithm on real-world DTMRI, we generate a synthetic fiber bundle in the bottom row of Fig. 1, which is again corrupted by additive noise. The result shows our method perfectly recovers the fine details of the structure, while smoothes out the noise.

Since for real-world DT-MR brain images, typically $128 \times 128 \times n(n>40)$, the number of image voxels is very large, it is time and space consuming to find the exact solution of our algorithm. We thus introduce the following approximation scheme to simplify the computations involved. Here we make use of the fact that the elements of the heat kernel $h_{t}(i, j)$ decay exponentially with the path length (the shortest distance) between nodes $i$ and $j$. As a result when we compute $I_{t}(j)$ for node $j$, we could ignore the effect of node $i$ if the path length between nodes $i$ and $j$ is larger than $\epsilon$. Hence, we can restrict our attention to voxels that are close to one another. To do this, we use a smaller $n_{1} \times n_{2} \times n_{3}$ volume window surrounding each voxel to construct a smaller sub-graph. We then use the heat kernel of this smaller sub-graph to calculate the Riemannian weighted mean $I_{t}(j)$ of the voxel $j$ at time $t$. 

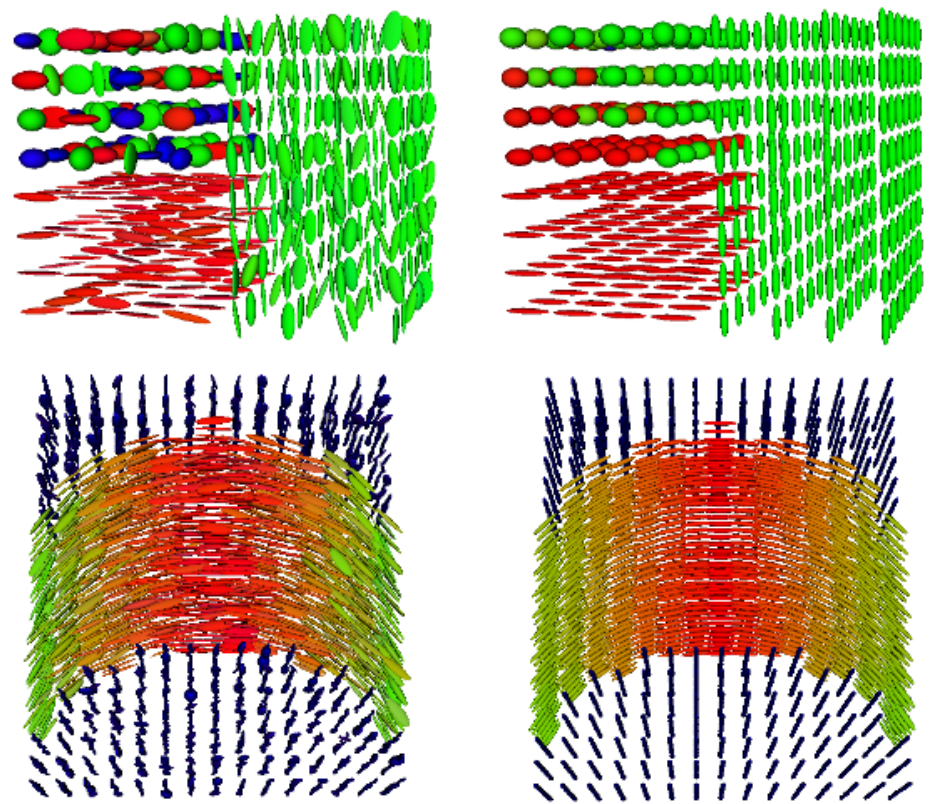

Fig. 1. Left column: noisy synthetic image. Right column: the corresponding regularized image. Both using parameters $r=5, \kappa_{I}=0.6, \kappa_{X}=2$.

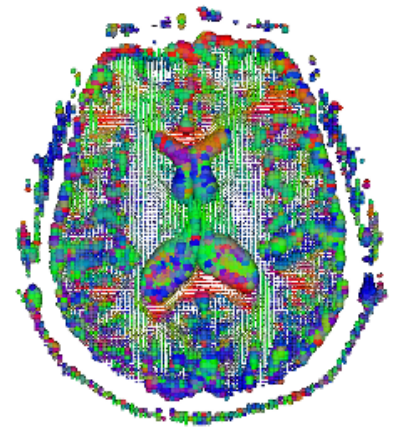

(a)

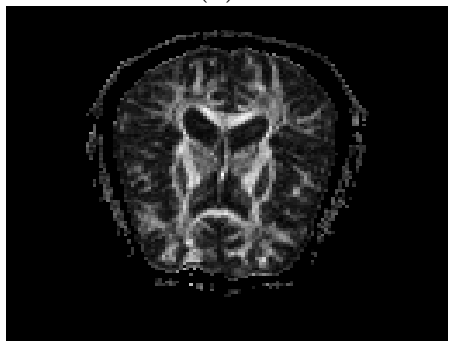

(c)

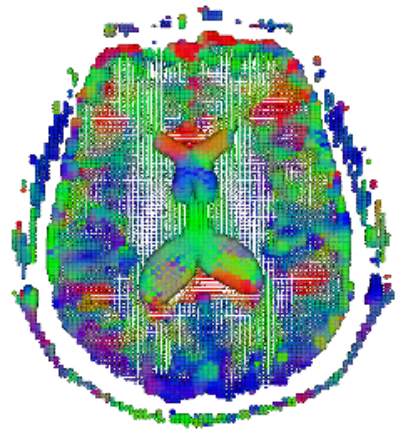

(b)

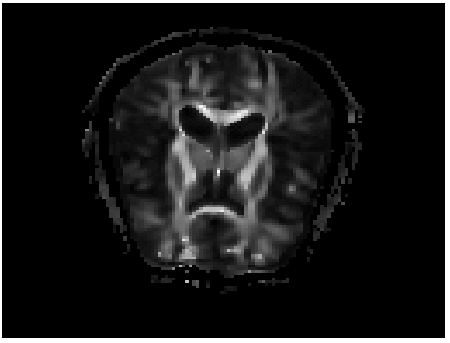

(d)

Fig. 2. (a) A slice of DT-MRI. (b) Graph regularized slice using approximate scheme by window size $10 \times 10 \times 5, r=4, \kappa_{I}=0.4, \kappa_{X}=1.7$. (c) and (d) are the corresponding fractional anisotropy map. 
Fig 2 shows a slice of the results of applying our method to a brain DT-MRI dataset of size $128 \times 128 \times 58$. The top row gives ellipsoid representation of the sample slice and it's regularized counterpart. The region with same color implies it's homogeneous structure inside. Thus, our method can sufficiently smooth each region. The bottom row gives the fractional anisotropy map [14] of the corresponding slice in the top row. The bright regions are potential places where fibers can be tracked. As the figure shows, discontinuities are preserved and the local coherence of the anisotropy map is enhanced after regularization.

\section{Conclusions}

We presented a graph-spectral method for diffusion tensor image regularization. The idea of our method is to represent DT-MRI using weighted attributed graphs, where diffusion across this graph-structure is captured by the heat kernel. DT-MRI regularization is effected by computing the Riemannian weighted mean using the heat kernel as its weights. The method can effectively eliminate the noise and preserve the coherence of the structures. Results on synthetic and real-world datasets proves the validation of the proposed algorithm.

\section{References}

1. C. Chefd'Hotel, D. Tschumperle, R. Deriche and O. Faugeras : Constrained flows of matrix-valued functions: Application to diffusion tensor regularization. In: Proc. of ECCV. (2002) 251-265

2. X. Pennec, P. Fillard, N. Ayache : A riemannian framework for tensor computing. International Journal of Computer Vision 66(1) (2006) 41-66

3. C. Poupon, J. Mangin, C. Clark, V. Frouin, J. Regis, D. Le Bihan and I. Bloch : Towards inference of human brain connectivity from $\mathrm{mr}$ diffusion tensor data. Medical Image Analysis 5 (2001) 1-15

4. D. Tschumperle and R. Deriche : Diffusion tensor regularization with constraints preservation. In: Proc. of CVPR. (2001) 948-953

5. O. Coulon, D. Alexander, S. Arridge : Diffusion tensor magnetic resonance image regularization. Medical Image Analysis 8 (2004) 47-67

6. Z. Wang, B. Vemuri, Y. Chen, T. Mareci : A constrained variational principle for direct estimation and smoothing of the diffusion tensor field from complex dwi. IEEE Trans. on Medical Imaging, 23(8) (2004) 930-939

7. Kondor, R., Lafferty, J.: Diffusion kernels on graphs and other discrete structures. In: Proc. of ICML. (2002)

8. P. Fletcher and S. Joshi : Principal geodesic analysis on symmetric spaces: Statistics of diffusion tensors. In: Proc. of CVAMIA, ECCV Workshop. (2004) 87-98

9. P. Batchelor, M. Moakher, D. Atkinson, F. Calamante and A. Connelly: A rigorous framework for diffusion tensor calculus. Mag. Res. in Medicine 53 (2005) 221-225

10. L. Skovgaard : A riemannian geometry of the multivariate normal model. Scandinavian Jour. of Statistics 11 (1984) 211-223

11. M. Moakher : A differential geometric approach to the geometric mean of symmetric positive-definite matrices. SIAM J. Mat. Anal. and App. 26 (2005) 735-747 
12. Shi, J., Malik, J.: Normalized cuts and image segmentation. IEEE Transactions on Pattern Analysis and Machine Intelligence 22(8) (2000) 888-905

13. Chung, F.: Spectral Graph Theory. American Mathmatical Society (1997)

14. C. Westin, S. Maier, H. Mamata, A. Nabavi, F. Jolesz and R. Kikinis: Processing and visualization for diffusion tensor mri. Medical Image Analysis 6 (2002) 93-108 